FIG. 2 Map of nodulation genes in two biovars of Rhizobium leguminosarum, a, by viciae, which infects pea, vetch, lentil and related plants; and $b$, by trifolii, which infects clovers. The genes have been established by transposon mutagenesis, sequencing and some protein product analysis ${ }^{4.5}$. Asterisks indicate positions in which various researchers believe further genes will soon be defined. Those genes designated 'common' are functionally interchangeable without an effect on host range; in particular, nodABC seem to stimulate host cell divisions and nodule formation, and are also required for root-hair deformation ${ }^{3}$. These common genes do not function well without accompanying host-specific nodulation genes, such as nodFE, which appear critical for proper root-hair curling and infection ${ }^{4.5}$. An example of a fine-structure host range gene is nodX, found in some but not all $R$. I. viciae; without nodX, R. I. viciae strains are restricted to nodulating only certain genotypes of pea ${ }^{5}$.

be important in this process. Whether changing the lectins expressed in other host plants can similarly affect infection by Rhizobium remains to be seen. The two bacteria which nodulate pea versus clover are more closely related than most pairs of Rhizobium. Those which are less closely related may have more dramatic limitations to nodulation on their heterologous hosts. It would not be surprising if different host/symbiont combinations in varying isolation evolved exclusivity and compatibility to different degrees and along slightly different avenues.

The molecular role of any receptor, of course, remains difficult to study until it is known to what it binds. This remains a sticky point in research on Rhizobiumlegume systems. If plant lectins are receptors for bacterial carbohydrates, it may be that the relevant sugars are sweetening up a surface component not identified to date. For example, researchers working on the genes which control several types of extracellular polysaccharides in Rhizobium find that eliminating or altering surface components such as the acidic exopolysaccharide $^{y}$ or the lipopolysaccharide ${ }^{10}$ prevents successful invasion, but allows formation of uninfected nodules in a correct host-specific manner. Therefore, these carbohydrates do not seem to correlate with host range, although it remains possible that there is redundant information in several surface determinants. Identification of specific bacterial molecules interacting with host components will be essential before the issue of how lectins affect the symbiosis is resolved.

The availability of transgenic roots with altered nodulation properties will allow genetically tailored microbes and plants to be paired with each other for specific mechanistic tests. It is of interest, for example, to test bacteria altered in known nodulation genes on transgenic roots with host-range changes that are complementary. Expression of lectin genes in heterologous backgrounds may help to determine exactly where the lectin is expressed and localized in transgenic plants, for example, and under what circumstances its functional properties are preserved. Previous studies on pea have shown that lectin binding of $R$. $l$. viciae can be assayed only under manganese limitation of the bacteria": do the transgenic clover/pea lectin roots bind their new $R$. l. viciae symbionts in this manner?

Finally, how are host-range functions coupled with the action of the common nodulation genes? Genes such as nod $A B C$ drive the plant to begin cell divisions, and are also required for root hair curling and invasion, but they do not have a productive effect on a host plant unless they are accompanied by the proper host range genes. Bacteria with normal nod $A B C$ genes, but lacking a host-range gene, for example, often cause the plant to exhibit incomplete or atypical root-hair deformations. If host-range genes are additive in effect, then perhaps they act by modifying a central function or functions provided by the common nod genes, increasing their efficiency. There is new experimental support for this model ${ }^{12}$. In addition, hostrange genes might also act by directing the location and timing of the action of Rhizobium on its target; by providing access for a molecular signal or a cell to a site of action; by actively modifying the bacteria's immediate molecular environment within the plant cell wall or membrane; by preventing a host defensive reaction; or by some combination of these. Given that the transfer of the pea lectin can change the responsiveness of clover roots to Rhizobium, it may be possible to test whether the function of this lectin is simply in attachment, or whether it acts through more complicated, possibly more active, mechanisms.

Sharon R. Long and David W. Ehrhardt are in the Department of Biological Sciences, Stanford University, Stanford, California 94305, USA.

1. Graham, T. L. Int. Rev. Cytol. Suppl. 13, 127-148 (1981)

2. Diaz, C. L., Melchers, L. S.,Hooykaas, P. J. J., Lugtenberg, B. J. J. \& Kijne, J. W. Nature 338, 579-581 (1989) Long, S. R. Cell 56, 203-214 (1989)

4. Rolfe, B. \& Gresshoff, P. A. Rev. Plant Physiol. Plant

5. Downie, J. A. \& Johnston, A. W. B. Plant Cell Environment 11, 403-412 (1989)

6. Dazzo, F. B. \& Gardiol, A in Genes used in Plant-Microbe Interactions (eds Verma, D. \& Hohn, T.) 3-31 (1984). Horvath, B. et al. Cell 46, 335-343 (1986)

7. Horvath, B. et al. Cell 46, 335-343 (1986).
8. Spaink, H. P. Wijfelman, C. A., Pees, E., Okker, R. J. H. Spaink, H. P., Wijffelman, C. A., Pees, E., Okker, R. J. H
\& Lugtenberg, B. J. J. Nature 328, 337-340 (1987). Finan T. M. et al. Cell 40, 869-877 (1985).

10. Cava, J. R., Elias, P. M. Turowski. D. A. \& Noel, K. D. J. Bact. 171, 8-15 (1989)

1. Kijne, J. W. Smit, G., Diaz, C. L. \& Lugtenberg, B. J. J. J. Bact. 170, 2994-3000 (1988).

2. Faucher, C. et al. J. Bact. 170, $5489-5499$ (1988). molec. Biol. 39, 297-319 (1988)

\section{Long sight}

LAST week Daedalus devised his 'Space Spider'. This ingenious spacecraft propels itself by melt-spinning and ejecting a fine optic fibre. Cunningly, the fibre is made of a lasing glass, excited by the sunlight of space. It amplifies light pulses as they traverse it, and forms an active optical communication link back to Earth.

Daedalus now reckons that his spaceborne light guide should be a powerful research tool in its own right. Thus it could form an astronomical interferometer of unprecedented resolution. The light from a stellar source spreads out in a wide wavefront. When two separate sections of the wavefront are recombined, traditionally by means of a pair of mirrors, the resulting interference pattern can be decoded into an image of the source. It is hard to give such an interferometer a mirror separation greater than about ten metres, so it can only just resolve the diameters of the nearer, bigger stars. But an active space-borne optic fibre could recombine sections of wavefront millions of kilometres apart.

Daedalus envisages a command spacecraft with two fibres extending out from it, their far ends anchored to two observation platforms. Each platform would use a stabilized telescope to image light from the chosen astronomical object onto the end of its fibre. The light from the two telescopes would flow down the fibres and interfere at the central command craft. If the orbits of the telescopes were chosen to vary the distance and angle between them, the changing interferogram would slowly accumulate an aperture-synthesized image of the object. A source a thousand light years away could be resolved to a few kilometres!

More boldly still, Daedalus wants to use his space-borne fibre to detect gravity waves. These energetic but elusive phenomena are emitted by massive moving objects like big, short-period binaries, collapsing neutron stars, supernovae, and black holes in the act of swallowing a star. Even gravity waves from the early days of the Universe may still be echoing around. They should shake an optical interferometer as they pass through it. Terrestrial interferometers have so far failed to detect them: but Daedalus's space-borne fibre interferometer should be mightily more sensitive. Its optical length would only change in second order, but the shift of the interference pattern should still be very clear. Gravity waves would also deform the fibre slightly as they passed through it. The birefringence thus induced would be tiny indeed - but a light pulse traversing a million kilometres of such a fibre should suffer detectable changes of polarization. Much exciting astrophysical mayhem, undetectable by optical or radio emission, should thus be revealed in all its gravity.

David Jones 